\title{
ARTICLE \\ Nrf2 activation ameliorates mechanical allodynia in paclitaxel-induced neuropathic pain
}

\author{
Ya-qun Zhou ${ }^{1}$, Dai-qiang Liu ${ }^{1}$, Shu-ping Chen ${ }^{1}$, Nan Chen ${ }^{1}$, Jia Sun ${ }^{1}$, Xiao-mei Wang ${ }^{1}$, Fei Cao ${ }^{2}$, Yu-ke Tian $^{1}$ and Da-wei Ye ${ }^{3}$
}

Paclitaxel-induced neuropathic pain (PINP) is refractory to currently used analgesics. Previous studies show a pivotal role of oxidative stress in PINP. Because the nuclear factor erythroid-2-related factor 2 (Nrf2) has been considered as the critical regulator of endogenous antioxidant defense, we here explored whether activation of Nrf2 could attenuate PINP. A rat model of PINP was established by intraperitoneal injection of paclitaxel $(2 \mathrm{mg} / \mathrm{kg}$ ) every other day with a final cumulative dose of $8 \mathrm{mg} / \mathrm{kg}$. Hind paw withdrawal thresholds (PWTs) in response to von Frey filament stimuli were used to assess mechanical allodynia. We showed that a single dose of Nrf2 activator, oltipraz $(10,50$, and $100 \mathrm{mg} / \mathrm{kg})$, dose-dependently attenuated established mechanical allodynia, whereas repeated injection of oltipraz ( $100 \mathrm{mg} \cdot \mathrm{kg}^{-1} \cdot \mathrm{d}^{-1}$, i.p. from $\mathrm{d} 14$ to $\mathrm{d} 18$ ) almost abolished the mechanical allodynia in PINP rats. The antinociceptive effect of oltipraz was blocked by pre-injection of Nrf2 inhibitor trigonelline (20 mg/kg, i.p.). Early treatment with oltipraz ( $100 \mathrm{mg} \cdot \mathrm{kg}^{-1} \cdot \mathrm{d}^{-1}$, i.p. from d 0 to $\mathrm{d}$ 6) failed to prevent the development of the PINP, but delayed its onset. Western blot and immunofluorescence analysis revealed that the expression levels of Nrf2 and HO-1 were significantly upregulated in the spinal cord of PINP rats. Repeated injection of oltipraz caused further elevation of the expression levels of Nrf2 and HO-1 in the spinal cord of PINP rats, which was reversed by pre-injection of trigonelline. These results demonstrate that oltipraz ameliorates PINP via activating Nrf2/HO-1-signaling pathway in the spinal cord.

Keywords: paclitaxel; neuropathic pain; oxidative stress; Nrf2; HO-1; oltipraz

Acta Pharmacologica Sinica (2020) 41:1041-1048; https://doi.org/10.1038/s41401-020-0394-6

\section{INTRODUCTION}

Paclitaxel is one of the most widely used chemotherapeutic drugs for the treatment of cancers, including ovarian cancer, breast cancer, lung cancer, and pancreatic cancer [1, 2]. However, treatment with even a single dose of paclitaxel may lead to peripheral neuropathic pain, which may persist long after its cessation [3, 4]. Paclitaxel-induced neuropathic pain (PINP) is characterized by numbness, tingling, and ongoing burning pain in the glove and stocking areas of the hands and feet $[5,6]$. Unfortunately, PINP is refractory to commonly used analgesic strategies, such as nonsteroidal anti-inflammatory drugs, antiepileptics, and antidepressants [7-9]. To date, no effective treatment is available to prevent or alleviate PINP due to the poor understanding of its mechanisms. Therefore, further studies are warranted to investigate novel therapeutic targets.

Previous studies have demonstrated a pivotal role of oxidative stress in PINP [10, 11]. Numerous reactive oxygen species (ROS) scavengers have been demonstrated to show potent analgesic effects against PINP [12-14]. Nuclear factor erythroid-2-related factor 2 (Nrf2) is considered to be a critical regulator of endogenous antioxidant defense $[15,16]$. Under physiological conditions, Nrf2 is restricted to the cytoplasm as part of the Kelch-like $\mathrm{ECH}$-associated protein 1 (Keap1)-Nrf2 complex. In response to oxidative stress, the Keap1-Nrf2 complex is dissociated. Nrf2 then translocates into the nucleus to bind antioxidant response elements, and regulates the transcription of downstream target genes, such as heme oxygenase-1 (HO-1), superoxide dismutase, glutathione reductase, and $\mathrm{NAD}(\mathrm{P}) \mathrm{H}$ :quinone oxidoreductase $1[17,18]$. However, the spinal role of the $\mathrm{Nrf2/HO}-1$-signaling pathway in PINP is largely unknown. In this study, we investigated the analgesic effect of oltipraz, an Nrf2 activator, in a rat model of PINP.

\section{MATERIALS AND METHODS}

Animals

Male Sprague-Dawley rats (starting weight of $200-220 \mathrm{~g}$, Tongji Medical College, Huazhong University of Science and Technology, Wuhan, China) were used in the present study. The rats were housed in a controlled environment (12-h light/dark cycle) with water and food available ad libitum. All experimental procedures were approved by the Animal Care and Use Committee of Huazhong University of Science and Technology.

Establishment of the paclitaxel-induced neuropathic pain model Paclitaxel (MedChemExpress, Monmouth Junction, NJ, USA) was dissolved in DMSO at a concentration of $50 \mathrm{mg} / \mathrm{mL}$ and stored as a stock solution at $-80^{\circ} \mathrm{C}$. The stock solution was then mixed with an equal volume of Tween 80 and diluted with sterile saline to a

\footnotetext{
${ }^{1}$ Department of Anesthesiology and Pain Medicine, Tongji Hospital, Tongji Medical College, Huazhong University of Science and Technology, Wuhan 430030, China; ${ }^{2}$ Department

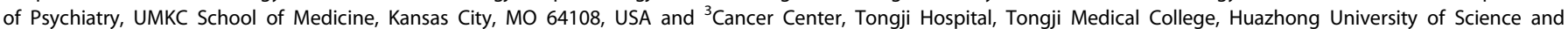
Technology, Wuhan 430030, China

Correspondence: Yu-ke Tian (yktian@tjh.tjmu.edu.cn) or Da-wei Ye (dy0711@gmail.com)
}

Received: 15 September 2019 Accepted: 5 March 2020

Published online: 19 March 2020 
1042

concentration of $2 \mathrm{mg} / \mathrm{mL}$ just before use. Paclitaxel ( $2 \mathrm{mg} / \mathrm{kg}$ ) was injected intraperitoneally (i.p.) on days $0,2,4$, and 6 at a final cumulative dose of $8 \mathrm{mg} / \mathrm{kg}$ as previously described to induce neuropathic pain $[19,20]$. Control rats received the same volume of vehicle, which consisted of $4 \%$ DMSO and $4 \%$ Tween 80 in sterile saline.

\section{Behavioral test}

Hind paw withdrawal thresholds (PWTs) in response to von Frey filament stimuli were used to assess mechanical allodynia as described previously [21, 22]. Briefly, animals were placed in individual chambers and allowed to acclimate for $30 \mathrm{~min}$. Starting with the 2-g filament, a series of von Frey filaments $(1,1.4,2,4,6$, 8,10 , and $15 \mathrm{~g}$ ) were applied to the midplantar area for $6 \mathrm{~s}$ per filament or until paw withdrawal. Abrupt paw withdrawal or licking in response to von Frey filament stimulation was considered a positive response. If a positive response was observed, the filament with the next lowest force was applied, and if no response occurred, the filament with the next highest force was used. The lowest force (in grams) required to elicit a positive response was recorded as the PWT. Since paclitaxel induces bilateral allodynia with no differences between the left and right hind paws, the PWT values of both paws were averaged. The experimenter who conducted the behavioral tests was blinded to the treatment conditions.

\section{Drug administration}

Oltipraz (MedChemExpress), a potent Nrf2 activator, was suspended in corn oil. Trigonelline hydrochloride (Sigma-Aldrich, Saint Louis, MO, USA), an Nrf2 inhibitor, was dissolved in sterile saline. The drug administration protocol is illustrated in Fig. 1. To determine whether a single dose of an Nrf2 activator can alleviate established PINP, oltipraz (10,50, or $100 \mathrm{mg} / \mathrm{kg}$, i.p.) was given on day 14 (d 14) after the first injection of paclitaxel. The behavioral test was conducted before oltipraz injection, and $0.5,1,2$, and $4 \mathrm{~h}$ after injection. To determine whether repetitive administration of an Nrf2 activator can reverse established PINP, oltipraz (100 mg/ $\mathrm{kg}$, i.p.) was given once daily from $\mathrm{d} 14$ to $\mathrm{d} 18$. The behavioral test was conducted at 13th day and $1 \mathrm{~h}$ after oltipraz injection each day. To determine whether the Nrf2 inhibitor can reverse the analgesic effect of oltipraz, trigonelline $(20 \mathrm{mg} / \mathrm{kg}$, i.p.) was given 30 min before oltipraz. The behavioral test was conducted before trigonelline injection, and $0.5,1,2$, and $4 \mathrm{~h}$ after oltipraz injection. To determine whether early treatment with an Nrf2 activator can suppress the development of PINP, oltipraz $(100 \mathrm{mg} / \mathrm{kg}$, i.p.) was given once daily from $\mathrm{d} 0$ to $\mathrm{d} 6$. The behavioral test was conducted on $\mathrm{d} 0$ before the first injection of paclitaxel, and on $\mathrm{d}$ $3, \mathrm{~d} 7, \mathrm{~d} 14$, and d 21 .

\section{Western blotting}

After the rats were anesthetized deeply with pentobarbital sodium $(100 \mathrm{mg} / \mathrm{kg}$, i.p.), the L4-L5 spinal cord was removed and homogenized in RIPA lysis buffer with PMSF, a protease inhibitor (Boster, Wuhan, China). The homogenates were centrifuged at $12,000 \mathrm{rpm}$ for $15 \mathrm{~min}$ at $4^{\circ} \mathrm{C}$. Then, the supernatants were collected, and the protein concentration was measured using a BCA protein assay kit (Boster). The samples were then heated at $95^{\circ} \mathrm{C}$ for $10 \mathrm{~min}$ in loading buffer. Equivalent amounts of proteins $(50 \mu \mathrm{g})$ were separated by electrophoresis on $10 \%$ sodium dodecyl sulfate-polyacrylamide gels and transferred to polyvinylidene fluoride membranes (PVDF, Millipore, Billerica, MA, USA). After blocking with $5 \%$ bovine serum albumin in Tris-buffered saline and Tween 20 (TBST) for $2 \mathrm{~h}$ at room temperature, the membranes were incubated with different antibodies: a rabbit anti-Nrf2 antibody (1:1000; 16396-1-AP, Proteintech, Chicago, IL, USA), a rabbit anti-HO-1 antibody (1:1000; A11919, ABclonal, Woburn, MA, USA), and a rabbit anti-glyceraldehyde-3-phosphate dehydrogenase (GAPDH) antibody (1:5000; 10494-1-AP, Proteintech), a Therapeutic effect of oltipraz (a single dose)

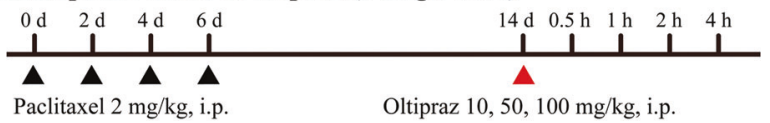

b Therapeutic effect of oltipraz (multiple doses)

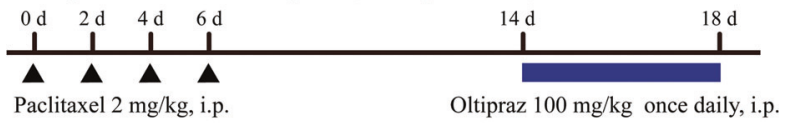

c Reversal effect of trigonelline, an Nrf2 inhibitor

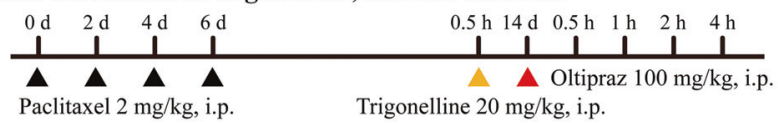

d Preventive effect of oltipraz

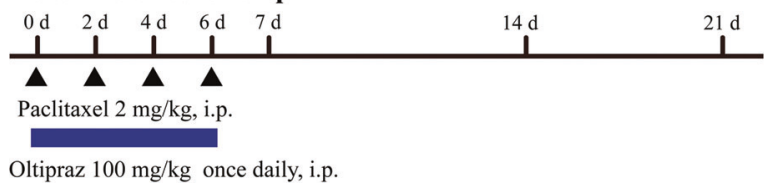

Fig. 1 Schematic illustration of the experimental design. a To determine whether a single dose of an Nrf2 activator can alleviate established PINP, oltipraz $(10,50$, or $100 \mathrm{mg} / \mathrm{kg}$, i.p.) was given on day 14 (d 14) after the first injection of paclitaxel. The behavioral test was conducted before oltipraz injection, and $0.5,1,2$, and $4 \mathrm{~h}$ after injection. b To determine whether repetitive injections of an Nrf2 activator can reverse established PINP, oltipraz (100 mg/kg, i.p.) was given once daily from d 14 to $\mathrm{d} 18$. The behavioral test was conducted on $\mathrm{d} 13$ and $1 \mathrm{~h}$ after oltipraz injection each day. c To determine whether an Nrf2 inhibitor can reverse the analgesic effect of oltipraz, trigonelline $(20 \mathrm{mg} / \mathrm{kg}$, i.p.) was given $30 \mathrm{~min}$ before oltipraz. The behavioral test was conducted before trigonelline injection, and $0.5,1,2$, and $4 \mathrm{~h}$ after oltipraz injection. d To determine whether early treatment with an Nrf2 activator can suppress the development of PINP, oltipraz $(100 \mathrm{mg} / \mathrm{kg}$, i.p.) was given once daily from $\mathrm{d} 0$ to $\mathrm{d} 6$. The behavioral test was conducted on $\mathrm{d} 0$ before the first injection of paclitaxel, and on $\mathrm{d} 3, \mathrm{~d} 7, \mathrm{~d} 14$, and $\mathrm{d} 21$

overnight at $4{ }^{\circ} \mathrm{C}$. After being washed with TBST, the membranes were incubated with horseradish peroxidase-conjugated goat anti-rabbit IgG (1:5000; A21020, Abbkine) for $2 \mathrm{~h}$ at room temperature. The bands were finally visualized using the SuperLumia ECL Plus HRP Substrate Kit (K22030; Abbkine, Wuhan, Hubei, China) and exposed using a computerized image analysis system (ChemiDoc XRS+, Bio-Rad Laboratories, Hercules, CA, USA). The intensity of the blots was quantified using the Bio-Rad ChemiDoc XRS+ system with Image Lab software (Bio-Rad Laboratories), normalized to the loading control GAPDH, and expressed as the fold of the control. The blot density of the control groups was set as 1 .

Immunofluorescence

After being anesthetized deeply with pentobarbital sodium (100 $\mathrm{mg} / \mathrm{kg}$, i.p.), the rats were intracardially perfused with phosphatebuffered saline (PBS) followed by $4 \%$ ice-cold paraformaldehyde in PBS. The L4-L5 spinal cord was removed, postfixed in $4 \%$ paraformaldehyde for $4 \mathrm{~h}$, and subsequently dehydrated in $30 \%$ sucrose solution for $48 \mathrm{~h}$ at $4{ }^{\circ} \mathrm{C}$. The samples were sectioned into $20-\mu$ m-thick slices on a cryostat (CM1900, Leica, Wiesbaden, Germany). After being washed in PBS, the sections were penetrated with $0.3 \%$ Triton X-100 for $15 \mathrm{~min}$, and blocked with $10 \%$ donkey serum for $1 \mathrm{~h}$ at room temperature. Then, the sections were incubated with a mixture of two primary antibodies overnight at $4{ }^{\circ} \mathrm{C}$, followed by a mixture of Alexa Fluor 488conjugated donkey anti-rabbit secondary antibody (1:100; 711547-003, Jackson ImmunoResearch, West Grove, PA, USA) and 
Alexa Fluor 594-conjugated donkey anti-mouse secondary antibody (1:200; 715-585-150, Jackson ImmunoResearch) or Alexa Fluor 488-conjugated donkey anti-rabbit secondary antibody (1:100, Jackson ImmunoResearch) and Alexa Fluor 594conjugated donkey anti-goat secondary antibody (1:200; 705585-003, Jackson ImmunoResearch). Specifically, to identify the cell types that expressed Nrf2 and HO-1, each of the antibodies was mixed with a mouse anti-neuronal nuclei (NeuN) antibody (neuronal marker, 1:100, ab104224, Abcam, Cambridge, UK), a mouse anti-glial fibrillary acidic protein (GFAP) antibody (astrocytic marker, 1:200, 3670, Cell Signaling Technology, Danvers, MA, USA), or a goat anti-lba1 antibody (microglial marker, 1:100, ab5076, Abcam). Images were then captured using a fluorescence microscope (DM2500, Leica).

\section{Statistical analysis}

The data are presented as the mean \pm SEM, and were analyzed by GraphPad Prism version 5.01 for Windows (GraphPad Software, San Diego, CA, USA). One-way analysis of variance (ANOVA) followed by Bonferroni post hoc test was used to analyze the Western blot data. Two-way repeated-measure ANOVA followed by Bonferroni post hoc test was used to analyze the behavioral data. $P<0.05$ was considered statistically significant.

\section{RESULTS}

Mechanical allodynia induced by intraperitoneal injections of paclitaxel

In the present study, we used a well-established rat model of PINP induced by intraperitoneal injections of paclitaxel on 4 alternating days $[23,24]$. PWT was examined on $d 0$ before the first injection of paclitaxel, and on d 3, d 7, d 14, and d 21, to evaluate the development of mechanical allodynia. No significant difference in the PWT was observed between vehicle-treated rats and PINP rats on $\mathrm{d} 0$ (Fig. 2). However, the PWT was significantly decreased from d 7 to $d 21(P<0.001$ compared with the vehicle group, $n=6$ in each group). In contrast, no significant change in the PWT was observed in vehicle-treated rats during the observation period. These results suggest that mechanical allodynia develops after intraperitoneal injections of paclitaxel.

Analgesic effect of oltipraz on established mechanical allodynia in PINP rats

To determine whether a single dose of an Nrf2 activator can alleviate established PINP, oltipraz (10,50, or $100 \mathrm{mg} / \mathrm{kg}$, i.p.) was given on d 14 after the first injection of paclitaxel. The behavioral test was conducted before oltipraz injection, and $0.5,1,2$, and $4 \mathrm{~h}$ after injection. No significant change in the PWT was observed

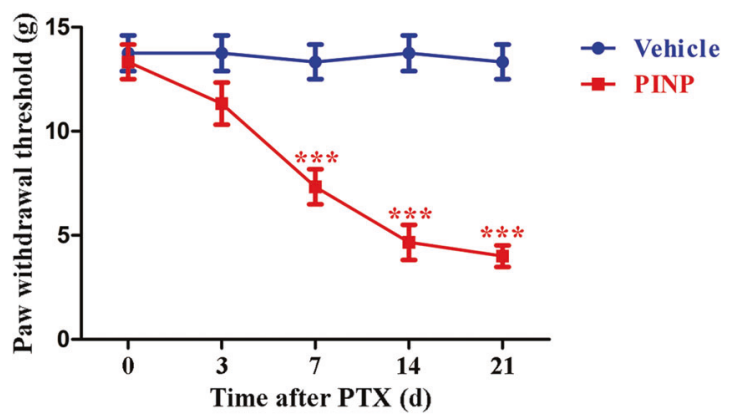

Fig. 2 Mechanical allodynia induced by intraperitoneal injections of paclitaxel. The PWT was significantly decreased from $d 7$ to $d 21$ after the first injection of paclitaxel ${ }^{* * *} P<0.001$ compared with the vehicle group, $n=6$ in each group). In contrast, no significant change in the PWT was observed in vehicle-treated rats during the observation period after a single intraperitoneal injection of oltipraz at a dose of $10 \mathrm{mg} / \mathrm{kg}$, compared with vehicle administration (Fig. $3 a, P>0.05$, $n=6$ in each group). However, oltipraz (50 and $100 \mathrm{mg} / \mathrm{kg}$ ) considerably increased the PWT of PINP rats; the change began at $0.5 \mathrm{~h}$, peaked at $1 \mathrm{~h}$, and lasted for $\sim 2 \mathrm{~h}(P<0.01, P<0.001$ compared with the vehicle group).
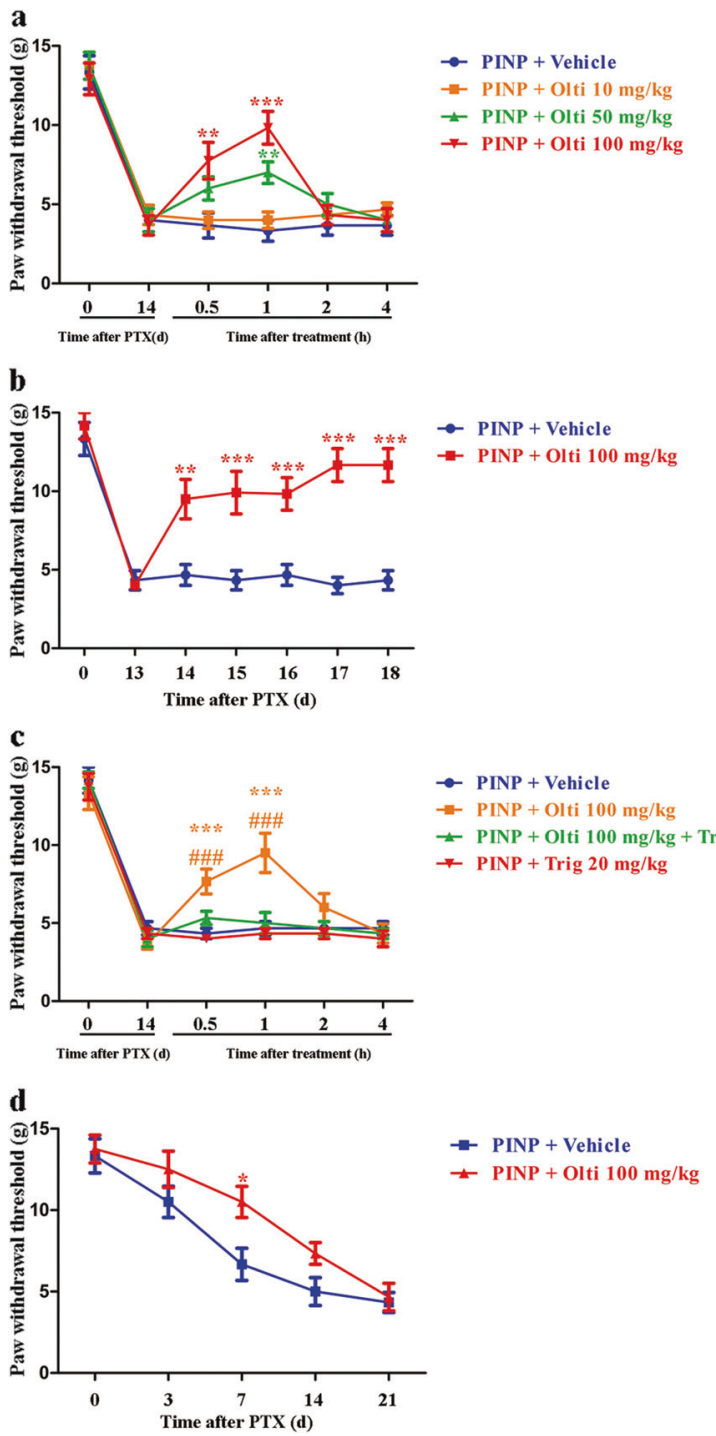

Fig. 3 Therapeutic and preventive effects of oltipraz on mechanical allodynia in PINP rats. a No significant change in the PWT was observed after a single intraperitoneal injection of oltipraz at a dose of $10 \mathrm{mg} / \mathrm{kg}$ compared with vehicle administration $(P>0.05)$. However, oltipraz (50 and $100 \mathrm{mg} / \mathrm{kg}$, i.p.) considerably increased the PWT of PINP rats; this change began at $0.5 \mathrm{~h}$, peaked at $1 \mathrm{~h}$, and lasted for $\sim 2 \mathrm{~h}\left({ }^{* *} P<0.01,{ }^{* * *} P<0.001\right.$ compared with the vehicle group, $n=6$ in each group). b Repeated injections of Olti $(100 \mathrm{mg} /$ $\mathrm{kg}$, i.p.) from d 14 to d 18 significantly reversed mechanical allodynia in PINP rats ${ }^{* * *} P<0.001$ compared with the vehicle group, $n=6$ in each group). c The analgesic effect of oltipraz (Olti, $100 \mathrm{mg} / \mathrm{kg}$, i.p.) in PINP was completely abolished by the Nrf2 inhibitor trigonelline (Trig, ${ }^{* *} P<0.001$ compared with the vehicle group, ${ }^{\# \#} P<0.001$ compared with the PINP $+100 \mathrm{mg} / \mathrm{kg}$ Olti $+20 \mathrm{mg} / \mathrm{kg}$ Trig group, $n=6$ in each group). d Early treatment with oltipraz (Olti, $100 \mathrm{mg} /$ $\mathrm{kg}$, i.p.) $30 \mathrm{~min}$ before the injection of paclitaxel from $\mathrm{d} 0$ to $\mathrm{d}$ 6 significantly elevated the PWT of PINP rats on $d 7$, but not on d 14 or d $21{ }^{*} P<0.05$ compared with the vehicle group, $n=6$ in each group) 
To determine whether repetitive injections of an Nrf2 activator can reverse established PINP, oltipraz (100 mg/kg, i.p.) was given once daily from $\mathrm{d} 14$ to $\mathrm{d} 18$. The behavioral test was conducted on $\mathrm{d} 13$ and $1 \mathrm{~h}$ after oltipraz injection each day. Repeated injections of oltipraz $(100 \mathrm{mg} / \mathrm{kg}$, i.p.) significantly reversed established mechanical allodynia in PINP rats (Fig. 3b, $P<0.001$ compared with the vehicle group, $n=6$ in each group). These data suggest that single and repeated injections of oltipraz have potent analgesic effects on PINP.

\section{Reversal effect of an Nrf2 inhibitor on the analgesic effect of oltipraz}

To determine whether an Nrf2 inhibitor can reverse the analgesic effect of oltipraz, trigonelline $(20 \mathrm{mg} / \mathrm{kg}$, i.p.) was given $30 \mathrm{~min}$ before oltipraz. The behavioral test was conducted before trigonelline injection, and $0.5,1,2$, and $4 \mathrm{~h}$ after oltipraz injection. As shown in Fig. 3c, the analgesic effect of oltipraz in PINP was entirely reversed by the Nrf2 inhibitor trigonelline $(P<0.001$ compared with the vehicle group, $P<0.001$ compared with the PINP $+100 \mathrm{mg} / \mathrm{kg}$ Olti $+20 \mathrm{mg} / \mathrm{kg}$ Trig group, $n=6$ in each group). This result suggests that oltipraz ameliorates established PINP by activating Nrf2.

\section{Preventive effect of early treatment with oltipraz on the} development of PINP

To determine whether early treatment with an Nrf2 activator can suppress the development of PINP, oltipraz $(100 \mathrm{mg} / \mathrm{kg}$, i.p.) was given once daily, 30 min before the injection of paclitaxel from d 0 to $\mathrm{d} 6$. The behavioral test was conducted on $\mathrm{d} 0$ before the first injection of paclitaxel, and on d 3, d 7, d 14, and d 21. As shown in Fig. 3d, the PWT was significantly elevated in oltipraz-treated PINP rats compared with vehicle-treated PINP rats on $d 7$, but not on $d$ 14 or d $21(P<0.05$ compared with the vehicle group, $n=6$ in each group). These results suggest that oltipraz does not completely prevent the development of mechanical allodynia induced by paclitaxel, but delays its onset.

Expression and cellular localization of Nrf2 and HO-1 in the spinal cord

Oltipraz ( $100 \mathrm{mg} / \mathrm{kg}$, i.p.) was given once daily from d 14 to $\mathrm{d} 18$. Trigonelline ( $20 \mathrm{mg} / \mathrm{kg}$, i.p.) was given $30 \mathrm{~min}$ before oltipraz. One hour after the final administration, the L4-L5 spinal cord was collected to detect the expression and cellular localization of Nrf2 and HO-1 in the spinal cord, using Western blotting and immunofluorescence. As shown in Figs. $4 a$ and $5 \mathrm{a}$, the spinal expression of Nrf2 and HO-1 was significantly increased in PINP rats $(P<0.05, P<0.01$ compared with the vehicle group, $n=6$ in each group). Moreover, oltipraz treatment further increased the spinal expression of $\mathrm{Nrf} 2$ and $\mathrm{HO}-1$ in PINP rats, which was reversed by the preadministration of trigonelline $(P<0.05, P<$ 0.001 compared with the vehicle group, $P<0.01, P<0.001$ compared with the oltipraz-treated group). The immunofluorescence data were consistent with the Western blotting results (Figs. $4 \mathrm{~b}$ and $5 \mathrm{~b}$ ). Moreover, both $\mathrm{Nrf2}$ and $\mathrm{HO}-1$ were localized in neurons (Figs. $4 \mathrm{~b}$ and 5b), and somewhat in astrocytes (Supplementary Fig. S1a and S2a) and microglia (Supplementary Fig. S1b and S2b) in the spinal cord dorsal horn. These results indicate that oltipraz may attenuate PINP by activating the Nrf2/ HO-1-signaling pathway.

\section{DISCUSSION}

In this study, we showed that (1) single and repeated injections of oltipraz had potent analgesic effects on established PINP, which were blocked by an Nrf2 inhibitor; (2) early treatment with oltipraz delayed the onset of PINP; (3) oltipraz further increased the expression of Nrf2 and HO- 1 in the spinal cords of PINP rats. Taken together, these results provide solid evidence that oltipraz ameliorates PINP by activating the Nrf2/HO-1-signaling pathway in the spinal cord.

As a frontline chemotherapeutic agent, paclitaxel often leads to the occurrence of PINP, which may result in the discontinuation of chemotherapy $[25,26]$. In addition, PINP usually persists long after the cessation of paclitaxel treatment, and is unfortunately refractory to commonly used analgesic strategies; thus, it affects the quality of life of cancer patients $[27,28]$. It has been reported that ROS scavengers significantly attenuate and prevent the development of PINP in animal models [12-14]. Moreover, various antioxidants have been demonstrated to show protective effects against PINP in clinical trials $[29,30]$. These studies suggest that oxidative stress plays a pivotal role in the development of PINP. The Nrf2/HO-1-signaling pathway is a promising therapeutic target for defense against oxidative stress [31, 32]. Previously, sulforaphane, another Nrf2 activator, was shown to possess potent analgesic effects [33, 34]. Moreover, an $\mathrm{HO}-1$ inducer exerts antinociceptive effects against neuropathic- and inflammatory pain [35-38]. However, the spinal role of the Nrf2/HO-1-signaling pathway in PINP remains largely unknown.

In the present study, we first determined the analgesic effect of the Nrf2 activator oltipraz in PINP. The behavioral results showed that a single dose of oltipraz significantly attenuated established mechanical allodynia in PINP rats, which was markedly blocked by the Nrf2 inhibitor trigonelline. The analgesic effect began at $0.5 \mathrm{~h}$, peaked at $1 \mathrm{~h}$, and lasted for $\sim 2 \mathrm{~h}$, suggesting that the analgesic effect was acute. Considering the time course of new protein synthesis, it is unlikely that increased expression of Nrf2 and HO-1 contributed to the acute analgesic effect of oltipraz. Therefore, we repeatedly administered oltipraz for 5 consecutive days, and a behavioral test was conducted $1 \mathrm{~h}$ after administration. We found that the repetitive administration of oltipraz considerably reversed established PINP. The acute analgesic effect of oltipraz might be attributed to increased activity of Nrf2 rather than increased expression of Nrf2. To determine whether early treatment with an Nrf2 activator can suppress the development of PINP, oltipraz (100 mg/kg, i.p.) was given once daily, $30 \mathrm{~min}$ before the injection of paclitaxel from d 0 to $d 6$. We found that the PWT was significantly elevated in oltipraz-treated PINP rats compared with vehicle-treated PINP rats on d 7, but not on d 14 or d 21 . Repetitive treatment with oltipraz may have resulted in a cumulative analgesic effect that led to an elevated PWT on $\mathrm{d} 7$. However, this cumulative analgesic effect may not have lasted for more than 1 week, leading to decreased PWTs on d 14 and d 21. Similarly, Kim et al. demonstrated that the intrathecal injection of sulforaphane either $5 \mathrm{~min}$ before or $1 \mathrm{~h}$ after L5 spinal nerve transection (SNT) significantly inhibits the induction of neuropathic pain [39]. However, sulforaphane administration on $d 7$ after surgery fails to suppress SNT-induced pain hypersensitivity. This discrepancy is mainly due to different animal models. Microglia are significantly activated at an early time point after SNT, which contributes to the induction of neuropathic pain following SNT. The analgesic effect of sulforaphane against SNTinduced neuropathic pain is primarily due to the inhibition of NADPH oxidase 2-derived ROS in spinal cord microglia. Therefore, sulforaphane may prevent the development of SNT-induced neuropathic pain, but fails to attenuate established pain hypersensitivity. However, microglia are not activated in the spinal cord after paclitaxel treatment, thus excluding their role in the pathogenesis of PINP [40]. In a very recent study, Díaz et al. claimed that oltipraz possesses analgesic and antidepressant effects in a mouse model of neuropathic pain induced by chronic constriction of the sciatic nerve [41]. They found that the inhibition of microglial activation and induction of the $\mathrm{Nrf} 2 / \mathrm{HO}-$ 1 -signaling pathway in the hippocampus and/or prefrontal cortex contributes to the protective effects of oltipraz.

$\mathrm{Nrf2}$ is one of the master regulators of endogenous antioxidant defense [42]. In response to oxidative stress, Nrf2 promotes the 

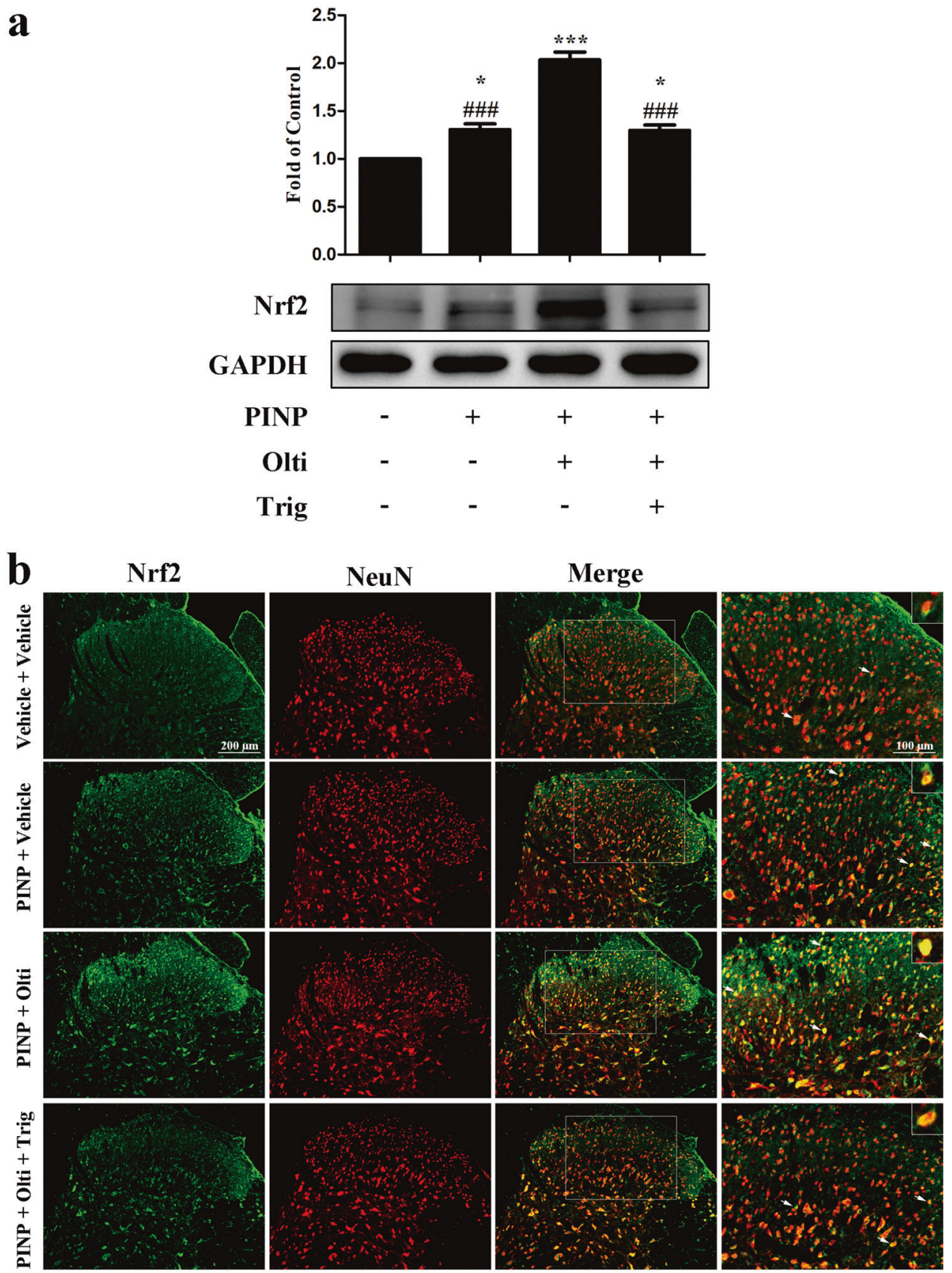

Fig. 4 Oltipraz increased the expression of Nrf2 in the spinal cords of PINP rats. a The spinal expression of Nrf2 was significantly increased in PINP rats $(* P<0.05$ compared with the vehicle group, $n=6$ in each group). Moreover, oltipraz treatment further increased the spinal expression of Nrf2 in PINP rats, which was reversed by the preadministration of trigonelline ${ }^{*} P<0.05,{ }^{*} * P<0.001$ compared with the vehicletreated group, ${ }^{\# \# \# ~} P<0.001$ compared with the oltipraz-treated group). b Nrf2 (green) was localized in neurons (as indicated by NeuN, a neuronal marker, red)

expression of a wide variety of antioxidant genes, including HO-1. Previous studies have demonstrated that the induction of $\mathrm{HO}-1$ expression has potential analgesic effects. Shen et al. found that both the intraperitoneal injection of HO-1 inducer CoPP and the intraspinal injection of an $\mathrm{HO}-1$-expressing lentivirus alleviates vincristine-induced neuropathic pain [43]. A similar analgesic effect of CoPP was demonstrated in a mouse model of L5 spinal nerve ligation (SNL)-induced neuropathic pain [44]. More importantly, Kim et al. found that the analgesic effect of the Nrf2 activator sulforaphane can be completely blocked by the $\mathrm{HO}-1$ inhibitor protoporphyrin [39]. These results indicate that $\mathrm{HO}-1$ plays a vital role in the analgesic effect of Nrf2 activators. We then examined the spinal expression and cellular localization of Nrf2 and HO-1 after oltipraz and paclitaxel treatments. To our knowledge, only a few studies have investigated the expression and distribution of Nrf2 and $\mathrm{HO}-1$ in the spinal cord under chronic pain conditions. Our results showed that the spinal expression of Nrf2 and HO-1 was significantly increased in PINP rats, indicating the activation of the endogenous antioxidant defense system. Furthermore, oltipraz treatment further increased the spinal expression of Nrf2 and HO-1 in PINP rats, which was reversed by the preadministration of trigonelline. However, decreased expression or no significant change in Nrf2 was reported in the DRGs of PINP rats [45-47]. The discrepancy might be attributed to differences in the tissues examined, and the time points of tissue collection. Interestingly, it seems that the expression of Nrf2 is 

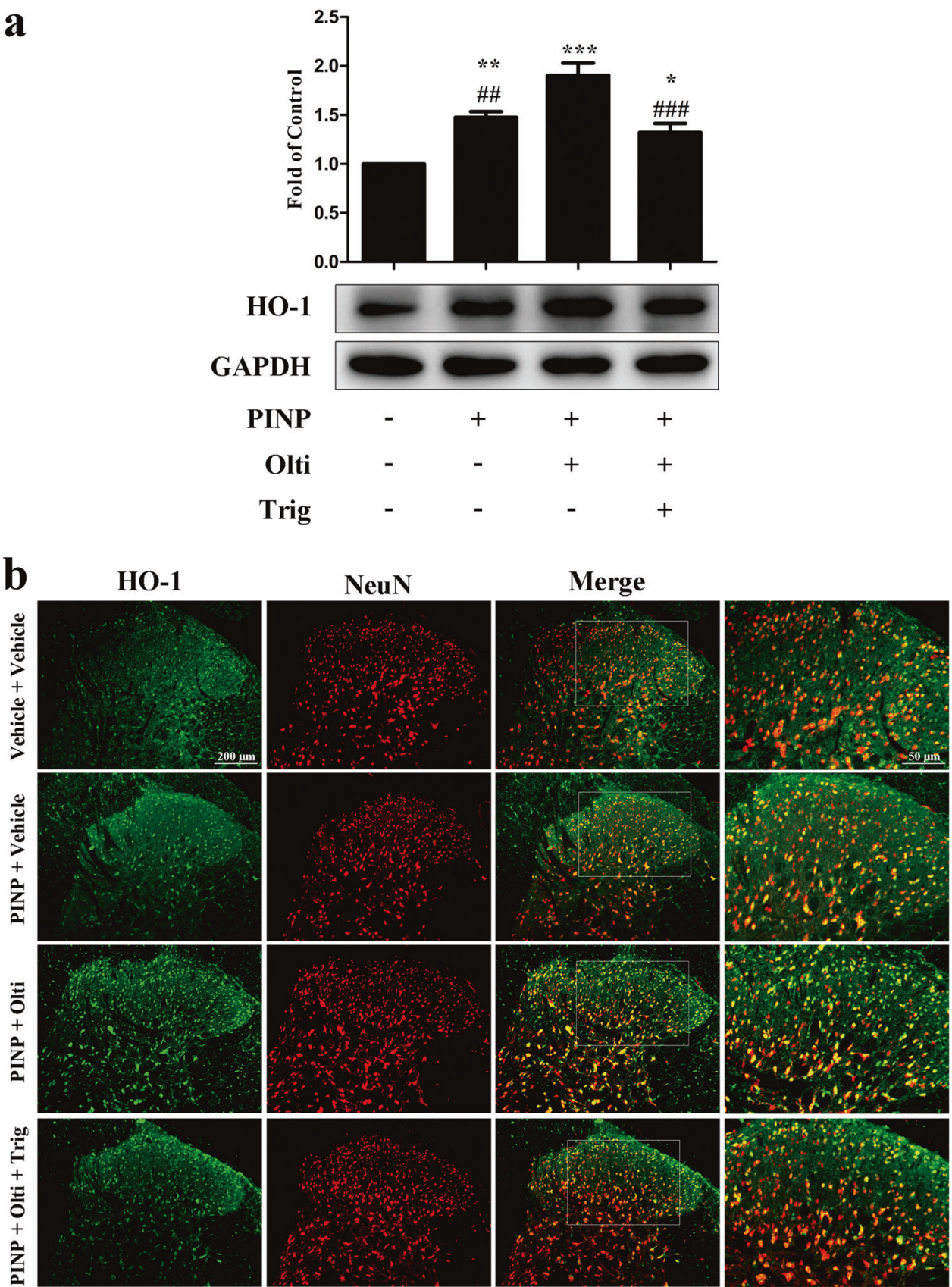

Fig. 5 Oltipraz increased the expression of HO-1 in the spinal cords of PINP rats. a The spinal expression of HO-1 was significantly increased in PINP rats $(* * P<0.01$ compared with the vehicle group, $n=6$ in each group). Moreover, oltipraz treatment further increased the spinal expression of HO-1 in PINP rats, which was reversed by the preadministration of trigonelline $(* P<0.05, * * * P<0.001$ compared with the vehicle group, ${ }^{\# \#} P<0.01,{ }^{\# \#} P<0.001$ compared with the oltipraz-treated group). b HO-1 (green) was localized in neurons (as indicated by NeuN, a neuronal marker, red)

altered in different stages and tissues in the PINP model. This suggests that Nrf2 may play distinct roles in particular stages and tissues, but this requires further study. These results indicate that oltipraz may attenuate PINP by activating the Nrf2/HO-1-signaling pathway in the spinal cord.

In summary, this study provides solid evidence that oltipraz significantly attenuates established PINP, and delays the onset of PINP, which can be reversed by an Nrf2 inhibitor. Moreover, the underlying mechanism of the analgesic effect of oltipraz in PINP might be due to induction of the Nrf2/HO-1-signaling pathway in the spinal cord. However, it remains unknown whether oltipraz alleviates PINP by directly reducing the level of ROS in the spinal cord, which is one of the limitations of this study. In addition, it would be interesting to determine whether ROS donors can reduce the analgesic effect of oltipraz, and whether oltipraz provides further benefits after animals are treated with antioxidants. Further studies are warranted to unravel these questions. It is worth mentioning that a variety of studies have indicated that oltipraz exerts cancer chemopreventive effects [48]. In addition, clinical trials have demonstrated the efficacy and safety of oltipraz in patients with nonalcoholic fatty liver disease or liver fibrosis $[49,50]$. However, Weerachayaphorn et al. found that oltipraz treatment exacerbates the severity of liver injury following bile duct ligation in mice [51]. Therefore, drug-related toxicity should 
not be ignored. Taken together, our results suggest that novel or currently available Nrf2 activators may become alternative therapeutic strategies for the management of PINP in the clinic.

\section{ACKNOWLEDGEMENTS}

This work was supported by grants from the National Natural Science Foundation of China (Grant Nos. 81873732, 81400917, 81371250, and 81571053).

\section{AUTHOR CONTRIBUTIONS}

YKT and DWY developed the concept of the study; YQZ and DQL designed the study; YQZ, SPC, FC, and JS provided experimental support; XMW and NC analyzed and interpreted the data; $Y Q Z$ wrote the paper.

\section{ADDITIONAL INFORMATION}

The online version of this article (https://doi.org/10.1038/s41401-020-0394-6) contains supplementary material, which is available to authorized users.

Competing interests: The authors declare no competing interests.

\section{REFERENCES}

1. Xie S, Ogden A, Aneja R, Zhou J. Microtubule-binding proteins as promising biomarkers of paclitaxel sensitivity in cancer chemotherapy. Med Res Rev. 2016;36:300-12.

2. Stage TB, Bergmann TK, Kroetz DL. Clinical pharmacokinetics of paclitaxel monotherapy: an updated literature review. Clin Pharmacokinet. 2018;57:7-19.

3. Starobova H, Vetter I. Pathophysiology of chemotherapy-induced peripheral neuropathy. Front Mol Neurosci. 2017;10:174.

4. Farquhar-Smith P. Chemotherapy-induced neuropathic pain. Curr Opin Support Palliat Care. 2011;5:1-7.

5. Massey RL, Kim HK, Abdi S. Brief review: chemotherapy-induced painful peripheral neuropathy (CIPPN): current status and future directions. Can J Anaesth. 2014;61:754-62.

6. Yazdani S, Abdi S. Brief review: pain management for cancer survivors: challenges and opportunities. Can J Anaesth. 2014;61:745-53.

7. Shinde SS, Seisler D, Soori G, Atherton PJ, Pachman DR, Lafky J, et al. Can pregabalin prevent paclitaxel-associated neuropathy?-An ACCRU pilot trial. Support Care Cancer. 2016;24:547-53.

8. Xiao W, Naso L, Bennett GJ. Experimental studies of potential analgesics for the treatment of chemotherapy-evoked painful peripheral neuropathies. Pain Med. 2008:9:505-17.

9. Kautio $A L$, Haanpaa $M$, Saarto $T$, Kalso $E$. Amitriptyline in the treatment of chemotherapy-induced neuropathic symptoms. J Pain Symptom Manag. 2008;35:31-9.

10. Shim HS, Bae C, Wang J, Lee KH, Hankerd KM, Kim HK, et al. Peripheral and central oxidative stress in chemotherapy-induced neuropathic pain. Mol Pain. 2019;15:1744806919840098.

11. Duggett NA, Griffiths LA, McKenna OE, de Santis V, Yongsanguanchai N, Mokori $\mathrm{EB}$, et al. Oxidative stress in the development, maintenance and resolution of paclitaxel-induced painful neuropathy. Neuroscience. 2016;333:13-26.

12. Kim HK, Zhang YP, Gwak YS, Abdi S. Phenyl N-tert-butylnitrone, a free radical scavenger, reduces mechanical allodynia in chemotherapy-induced neuropathic pain in rats. Anesthesiology. 2010;112:432-9.

13. Kim HK, Hwang SH, Abdi S. Tempol ameliorates and prevents mechanical hyperalgesia in a rat model of chemotherapy-induced neuropathic pain. Front Pharmacol. 2016;7:532.

14. Fidanboylu M, Griffiths LA, Flatters SJ. Global inhibition of reactive oxygen species (ROS) inhibits paclitaxel-induced painful peripheral neuropathy. PLoS ONE. 2011;6:e25212.

15. Catanzaro E, Calcabrini C, Turrini E, Sestili P, Fimognari C. Nrf2: a potential therapeutic target for naturally occurring anticancer drugs? Expert Opin Ther Targets. 2017;21:781-93

16. Silva-Islas CA, Maldonado PD. Canonical and non-canonical mechanisms of Nrf2 activation. Pharmacol Res. 2018;134:92-9.

17. Raghunath A, Sundarraj K, Nagarajan R, Arfuso F, Bian J, Kumar AP, et al. Antioxidant response elements: discovery, classes, regulation and potential applications. Redox Biol. 2018;17:297-314.

18. Zhao GJ, Hou N, Cai SA, Liu XW, Li AQ, Cheng CF, et al. Contributions of Nrf2 to puerarin prevention of cardiac hypertrophy and its metabolic enzymes expression in rats. J Pharmacol Exp Ther. 2018;366:458-69.
19. Li Y, Tatsui CE, Rhines LD, North RY, Harrison DS, Cassidy RM, et al. Dorsal root ganglion neurons become hyperexcitable and increase expression of voltagegated T-type calcium channels (Cav3.2) in paclitaxel-induced peripheral neuropathy. Pain. 2017;158:417-29.

20. Kim HK, Kwon JY, Yoo C, Abdi S. The analgesic effect of rolipram, a phosphodiesterase 4 inhibitor, on chemotherapy-induced neuropathic pain in rats. Anesth Analg. 2015;121:822-8.

21. Zhou YQ, Chen SP, Liu DQ, Manyande A, Zhang W, Yang SB, et al. The role of spinal $G A B A B$ receptors in cancer-induced bone pain in rats. J Pain 2017;18:933-46.

22. Zhou YQ, Liu DQ, Chen SP, Sun J, Zhou XR, Rittner $H$, et al. Reactive oxygen species scavengers ameliorate mechanical allodynia in a rat model of cancerinduced bone pain. Redox Biol. 2018;14:391-97.

23. Kim E, Hwang SH, Kim HK, Abdi S, Kim HK. Losartan, an angiotensin II type 1 receptor antagonist, alleviates mechanical hyperalgesia in a rat model of chemotherapy-induced neuropathic pain by inhibiting inflammatory cytokines in the dorsal root ganglia. Mol Neurobiol. 2019;56:7408-19.

24. Li Y, North RY, Rhines LD, Tatsui CE, Rao G, Edwards DD, et al. DRG voltage-gated sodium channel 1.7 is upregulated in paclitaxel-induced neuropathy in rats and in humans with neuropathic pain. J Neurosci. 2018;38:1124-36.

25. Kim HK, Hwang SH, Oh E, Abdi S. Rolipram, a selective phosphodiesterase 4 inhibitor, ameliorates mechanical hyperalgesia in a rat model of chemotherapyinduced neuropathic pain through inhibition of inflammatory cytokines in the dorsal root ganglion. Front Pharmacol. 2017;8:885.

26. Luo J, Bavencoffe A, Yang P, Feng J, Yin S, Qian A, et al. Zinc inhibits TRPV1 to alleviate chemotherapy-induced neuropathic pain. J Neurosci. 2018;38:474-83.

27. Hopkins HL, Duggett NA, Flatters SJL. Chemotherapy-induced painful neuropathy: pain-like behaviours in rodent models and their response to commonly used analgesics. Curr Opin Support Palliat Care. 2016;10:119-28.

28. Nie B, Liu C, Bai X, Chen X, Wu S, Zhang S, et al. AKAP150 involved in paclitaxelinduced neuropathic pain via inhibiting CN/NFAT2 pathway and downregulating IL-4. Brain Behav Immun. 2018;68:158-68.

29. Ghoreishi Z, Esfahani A, Djazayeri A, Djalali M, Golestan B, Ayromlou H, et al. Omega-3 fatty acids are protective against paclitaxel-induced peripheral neuropathy: a randomized double-blind placebo controlled trial. BMC Cancer. 2012;12:355.

30. Argyriou AA, Chroni E, Koutras A, Iconomou G, Papapetropoulos S, Polychronopoulos $\mathrm{P}$, et al. Preventing paclitaxel-induced peripheral neuropathy: a phase II trial of vitamin E supplementation. J Pain Symptom Manag. 2006;32:237-44.

31. Zhang R, Xu M, Wang Y, Xie F, Zhang G, Qin X. Nrf2-a promising therapeutic target for defensing against oxidative stress in stroke. Mol Neurobiol 2017;54:6006-17.

32. Loboda A, Damulewicz M, Pyza E, Jozkowicz A, Dulak J. Role of Nrf2/HO-1 system in development, oxidative stress response and diseases: an evolutionarily conserved mechanism. Cell Mol Life Sci. 2016;73:3221-47.

33. Redondo A, Chamorro PAF, Riego G, Leanez S, Pol O. Treatment with sulforaphane produces antinociception and improves morphine effects during inflammatory pain in mice. J Pharmacol Exp Ther. 2017;363:293-302.

34. Ferreira-Chamorro P, Redondo A, Riego G, Leanez S, Pol O. Sulforaphane inhibited the nociceptive responses, anxiety- and depressive-like behaviors associated with neuropathic pain and improved the anti-allodynic effects of morphine in mice. Front Pharmacol. 2018;9:1332.

35. Carcole M, Castany S, Leanez S, Pol O. Treatment with a heme oxygenase 1 inducer enhances the antinociceptive effects of micro-opioid, delta-opioid, and cannabinoid 2 receptors during inflammatory pain. J Pharmacol Exp Ther. 2014;351:224-32.

36. Riego G, Redondo A, Leanez S, Pol O. Mechanism implicated in the anti-allodynic and anti-hyperalgesic effects induced by the activation of heme oxygenase $1 /$ carbon monoxide signaling pathway in the central nervous system of mice with neuropathic pain. Biochem Pharmacol. 2018;148:52-63.

37. Hervera A, Gou G, Leanez S, Pol O. Effects of treatment with a carbon monoxidereleasing molecule and a heme oxygenase 1 inducer in the antinociceptive effects of morphine in different models of acute and chronic pain in mice. Psychopharmacology. 2013;228:463-77.

38. Hervera $A$, Leanez $S$, Motterlini $R$, Pol $O$. Treatment with carbon monoxidereleasing molecules and an $\mathrm{HO}-1$ inducer enhances the effects and expression of micro-opioid receptors during neuropathic pain. Anesthesiology. 2013; 118:1180-97.

39. Kim D, You B, Jo EK, Han SK, Simon MI, Lee SJ. NADPH oxidase 2-derived reactive oxygen species in spinal cord microglia contribute to peripheral nerve injuryinduced neuropathic pain. Proc Natl Acad Sci USA. 2010;107:14851-6.

40. Zhang $H$, Yoon SY, Zhang $H$, Dougherty PM. Evidence that spinal astrocytes but not microglia contribute to the pathogenesis of paclitaxel-induced painful neuropathy. J Pain. 2012;13:293-303. 
1048

41. Diaz AF, Polo S, Gallardo N, Leanez S, Pol O. Analgesic and antidepressant effects of oltipraz on neuropathic pain in mice by modulating microglial activation. J Clin Med. 2019;8:1-20.

42. Schmidlin CJ, Dodson MB, Madhavan L, Zhang DD. Redox regulation by NRF2 in aging and disease. Free Radic Biol Med. 2019;134:702-7.

43. Shen Y, Zhang ZJ, Zhu MD, Jiang BC, Yang T, Gao YJ. Exogenous induction of HO1 alleviates vincristine-induced neuropathic pain by reducing spinal glial activation in mice. Neurobiol Dis. 2015;79:100-10.

44. Liu X, Zhang Z, Cheng Z, Zhang J, Xu S, Liu H, et al. Spinal heme oxygenase-1 (HO-1) exerts antinociceptive effects against neuropathic pain in a mouse model of L5 spinal nerve ligation. Pain Med. 2016;17:220-9.

45. Sun H, Guo X, Wang Z, Wang P, Zhang Z, Dong J, et al. Alphalipoic acid prevents oxidative stress and peripheral neuropathy in nab-paclitaxel-treated rats through the Nrf2 signalling pathway. Oxid Med Cell Longev. 2019;2019:3142732.

46. Singh J, Saha L, Singh N, Kumari P, Bhatia A, Chakrabarti A. Study of nuclear factor-2 erythroid related factor-2 activator, berberine, in paclitaxel induced peripheral neuropathy pain model in rats. J Pharm Pharmacol. 2019;71:797-805.
47. Zhao X, Liu L, Wang Y, Wang G, Zhao Y, Zhang Y. Electroacupuncture enhances antioxidative signal pathway and attenuates neuropathic pain induced by chemotherapeutic paclitaxel. Physiol Res. 2019;68:501-10.

48. Choi SH, Kim YM, Lee JM, Kim SG. Antioxidant and mitochondrial protective effects of oxidized metabolites of oltipraz. Expert Opin Drug Metab Toxicol. 2010;6:213-24.

49. Kim SG, Kim YM, Choi JY, Han JY, Jang JW, Cho SH, et al. Oltipraz therapy in patients with liver fibrosis or cirrhosis: a randomized, doubleblind, placebo-controlled phase II trial. J Pharm Pharmacol. 2011;63: 627-35.

50. Kim W, Kim BG, Lee JS, Lee CK, Yeon JE, Chang MS, et al. Randomised clinical trial: the efficacy and safety of oltipraz, a liver $X$ receptor alpha-inhibitory dithiolethione in patients with non-alcoholic fatty liver disease. Aliment Pharmacol Ther. 2017;45:1073-83.

51. Weerachayaphorn J, Luo Y, Mennone A, Soroka CJ, Harry K, Boyer JL. Deleterious effect of oltipraz on extrahepatic cholestasis in bile duct-ligated mice. J Hepatol. 2014;60:160-6. 